\title{
On the analytic solution of the Balitsky-Kovchegov evolution equation
}

\section{Sergey Bondarenko and Alex Prygarin}

Department of Physics, Ariel University, Ramat Ha-Golan St. 35, Ariel 40700, Israel

E-mail: sergeyb@ariel.ac.il, alexanderp@ariel.ac.il

ABSTRACT: The study presents an analytic solution of the Balitsky-Kovchegov (BK) equation in a particular kinematics. The solution is written in the momentum space and based on the eigenfunctions of the truncated Balitsky-Fadin-Kuraev-Lipatov (BFKL) equation in the gauge adjoint representation, which was used for calculation of the Regge (Mandelstam) cut contribution to the planar helicity amplitudes. We introduce an eigenfunction of the singlet BFKL equation constructed of the adjoint eigenfunction multiplied by a factor, which restores the dual conformal symmetry present in the adjoint and broken in the singlet BFKL equations. The proposed analytic BK solution correctly reproduces the initial condition and the high energy asymptotics of the scattering amplitude.

KeYwords: Deep Inelastic Scattering, QCD

ArXiv EPRINT: 1503.05437 


\section{Contents}

1 Introduction 1

2 Solution of the BK equation $\quad 2$

$\begin{array}{llr}3 & \text { Discussions } & 8\end{array}$

A Calculation of $\left.\mathcal{N}(\mathrm{k}, \mathrm{q})\right|_{Y \rightarrow \infty} \quad 9$

\section{Introduction}

The power growth of the Balitsky-Fadin-Kuraev-Lipatov (BFKL) [1-3] amplitude contradicts the unitarity condition and should be modified. In the pioneering paper of Gribov, Levin and Ryskin (GLR) [4] the authors introduced a gluon saturation, meaning that the gluon density grows at large energy resulting in an overlap of the gluon states which tams growth of the amplitude with increase of energy. This mechanism introduces a new scale commonly referred to as a saturation scale $Q_{s}^{2}$. The GLR equation was first to account for non-linear effects for describing the physics of the Deep Inelastic Scattering (DIS). The GLR equation has a linear term representing BFKL type ladder diagrams, but also it includes a non-linear term, which stands for so-called fan diagrams describing the pomeron splitting into two pomerons. The GLR equation was formulated in double logarithmic kinematics, where one resums powers of logarithm of energy and typical transverse momentum. This kinematics is more restricted with respect to the BFKL multi-Regge kinematics, where the energy of the colliding particles is much larger than any transverse momentum. A decade after formulation of the GLR equation the unitarization corrections in the Regge kinematics were taken into account in the further generalization of the BFKL equation in the Wilson line formalism by Balitsky [5] and soon after that independently by Kovchegov [6, 7] in the color dipole approach [8-12]. The Balitsky chain reduced to the equation derived by Kovchegov in the limit of infinite number of colors. The resulting Balitsky-Kovchegov (BK) equation has a relatively simple form in the transverse coordinate space where the integral Kernel of the linear and the non-linear terms are happened to be the same and have a simple interpretation of splitting of the color dipole into two dipoles. Despite numerous attempts during almost two decades the full analytic solution to the BK equation is still to be found. The linear part of the BK equation corresponds to the BFKL evolution, which was completely solved exploiting conformal symmetry of the BFKL Kernel in the space of transverse coordinates. However, the direct substitution of the BFKL eigenfunctions in the coordinate space to the BK equation does not lead to significant simplifications in solving the BK equation. It was recently proposed that BFKL equation in color adjoint representation can be solved for non-zero transverse momentum using the eigenfunction in the 
momentum space. This was done for calculating the Regge (Mandelstam) cut contribution to the planar helicity amplitudes in Regge kinematics. A resulting series of publications confirmed this calculations to be correct by performing analytic continuation followed by Regge limit of the so-called remainder function of the Bern-Dixon-Smirnov (BDS) [13] scattering amplitude. The analytic solution of the color adjoint BFKL was also useful for calculating Regge cut corrections to the planar amplitudes of the next-to-maximally helicity violating (NMHV) configuration of the scattered particles [14]. The Moebius representation of the adjoint BFKL equation was calculated in ref. $[15,16]$.

In the present study we build a general form of the analytic solution to the BK equation (see eq. (2.10)) based on the eigenfunctions of the color adjoint BFKL equation. The difference between the eigenfunctions of the adjoint BFKL in eq. (2.6) and the eigenfunctions of the singlet BFKL in eq. (2.7) is mainly in the proper normalization condition leading to a factor, which is related to a shift the argument of the BFKL eigenvalue by $1 / 2$ with respect the eigenvalue of adjoint BFKL. This factor is needed to restore the conformal symmetry in the momentum space of dual coordinates $p_{i}=z_{i}-z_{i+1}, i=1,2$ present in the adjoint BFKL and broken in the singlet case. The dual conformal symmetry is believed to be a sign of integrability in planar amplitudes and is supposed to be present in the integrable singlet BFKL equation.

Next, assuming a particular dependence of the impact factor on the transferred momentum in eq. (2.30) we find a closed analytic solution of the Balitsky-Kovchegov equation in this particular kinematics. Finally, we check that our solution correctly reproduces the initial condition and the high energy asymptotics of the scattering amplitude. Some details of the calculations are presented in the appendix.

\section{Solution of the BK equation}

The Balitsky-Kovchegov (BK) [5-7] equation describes the energy evolution of the imaginary part of the scattering amplitude $N(\mathbf{x}, \mathbf{y})$ as follows

$$
\partial_{Y} N(\mathbf{x}, \mathbf{y})=\frac{\bar{\alpha}_{s}}{2 \pi} \int \frac{d^{2} \mathbf{z}(\mathbf{x}-\mathbf{y})^{2}}{(\mathbf{x}-\mathbf{z})^{2}(\mathbf{z}-\mathbf{y})^{2}}(N(\mathbf{x}, \mathbf{z})+N(\mathbf{z}, \mathbf{y})-N(\mathbf{x}, \mathbf{y})-N(\mathbf{x}, \mathbf{z}) N(\mathbf{z}, \mathbf{y})) .
$$

The BK equation is written in terms of 't Hooft coupling

$$
\bar{\alpha}_{s}=\frac{\alpha_{s} N_{c}}{\pi}
$$

and the rapidity

$$
Y=\ln \left(\frac{s}{s_{0}}\right)
$$

where $s$ is the square of the total energy of the scattering particles and $s_{0}$ is some energy scale. Numerous attempts of solving BK equation in coordinate space suggest us looking for analytic solution in other representations. It is natural to start with momentum space using a notation of ref. [17] for the corresponding Fourier transform

$$
\mathcal{N}(\mathbf{k}, \mathbf{q})=\int \frac{d^{2} \mathbf{x}}{2 \pi} \frac{d^{2} \mathbf{y}}{2 \pi} e^{i \mathbf{k x}} e^{i(\mathbf{q}-\mathbf{k}) \mathbf{y}} \frac{N(\mathbf{x}, \mathbf{y})}{(\mathbf{x}-\mathbf{y})^{2}}
$$


and write the Balitsky-Kovchegov equation in the momentum space as follows

$$
\begin{aligned}
\partial_{Y} \mathcal{N}(\mathbf{k}, \mathbf{q})= & \frac{\bar{\alpha}_{s}}{\pi} \int \frac{d^{2} \mathbf{k}^{\prime}}{\left(\mathbf{k}-\mathbf{k}^{\prime}\right)^{2}}\left(\mathcal{N}\left(\mathbf{k}^{\prime}, \mathbf{q}\right)-\frac{1}{4}\left[\frac{(\mathbf{q}-\mathbf{k})^{2}}{\left(\mathbf{q}-\mathbf{k}^{\prime}\right)^{2}}+\frac{\mathbf{k}^{2}}{\mathbf{k}^{\prime 2}}\right] \mathcal{N}(\mathbf{k}, \mathbf{q})\right) \\
& -\frac{\bar{\alpha}_{s}}{2 \pi} \int d^{2} \mathbf{k}^{\prime} \mathcal{N}\left(\mathbf{k}, \mathbf{k}^{\prime}\right) \mathcal{N}\left(\mathbf{k}-\mathbf{k}^{\prime}, \mathbf{q}-\mathbf{k}^{\prime}\right) .
\end{aligned}
$$

Before considering the non-linear evolution we go back to the linear BFKL equation, which can be solved in two different ways; in the coordinate and in the momentum spaces. Both solutions are well known and usually addressed to as $q=0$ solution (momentum space) and $q \neq 0$ solution (coordinate space). Those two were thoroughly discussed in numerous studies and we do not focus on them for the sake of brevity. For more details on these two solutions the reader is referred to review texts [18-22]. This is true for the BFKL equation projected on the color singlet state of two interacting reggeized gluons. However, if one considers color adjoint BFKL with some infra-red divergent parts removed, there is another $q \neq 0$ solution in the momentum space expressed through the adjoint BFKL eigenfunctions ${ }^{1}$

$$
f_{\nu, n}(k, q)=\left(\frac{k}{q-k}\right)^{i \nu+\frac{n}{2}}\left(\frac{k^{*}}{q^{*}-k^{*}}\right)^{i \nu-\frac{n}{2}}
$$

which were used in a serious of publications [23-30] for calculations of the Regge (Mandelstam) cut contribution to the helicity amplitudes in maximally supersymmetric theory. Its solution was written in terms of $f_{\nu, n}(k, q)$ and a corresponding eigenvalue of the adjoint BFKL equation of shifted argument with respect to the singlet BFKL eigenvalue. The corresponding adjoint BFKL Green function was investigated in ref. [31].

In the present study we consider a non-linear generalization of the linear BFKL equation, namely the Balitsky-Kovchegov (BK) evolution equation. We introduce an ansatz for the BK solution in a particular kinematic regime, which can be written in terms of functions similar to $f_{\nu, n}(k, q)$. We slightly redefine the "adjoint" $f_{\nu, n}(k, q)$ to account for a proper normalization of the singlet BFKL as well as a minus sign, which happens to be important for the non-linear term of the BK equation as follows

$$
F_{\nu, n}(k, q)=\frac{1}{\sqrt{2} \pi} \frac{1}{|k||k-q|}\left(\frac{k}{k-q}\right)^{i \nu+\frac{n}{2}}\left(\frac{k^{*}}{k^{*}-q^{*}}\right)^{i \nu-\frac{n}{2}}=\frac{1}{\sqrt{2} \pi} \frac{(-1)^{n}}{|k||k-q|} f_{\nu, n}(k, q) .
$$

The function in eq. (2.7) solves the leading order singlet BFKL equation as we show below. The major difference between the adjoint and singlet eigenfunctions is the factor $\frac{1}{|k||k-q|}$, which restores the dual conformal symmetry of the singlet BFKL equation. This analysis is beyond the scope of the present study and will be published by us elsewhere.

The corresponding orthogonality condition is given by

$$
\int d^{2} \mathbf{k} F_{\nu, n}^{*}(k, q) F_{\nu^{\prime}, n^{\prime}}(k, q)=\frac{\delta\left(\nu-\nu^{\prime}\right) \delta_{n, n^{\prime}}}{|q|^{2}}
$$

and the completeness condition reads

$$
\sum_{n=-\infty}^{\infty} \int_{-\infty}^{\infty} d \nu F_{\nu, n}^{*}\left(k^{\prime}, q\right) F_{\nu, n}(k, q)=\frac{\delta^{2}\left(k-k^{\prime}\right)}{|q|^{2}} .
$$

\footnotetext{
${ }^{1}$ Here we use complex coordinates defined by $k=k_{x}+i k_{y}, k^{*}=k_{x}-i k_{y}$
} 
Then we can write the analytic solution of the Balitsky-Kovchegov equation in the momentum space as follows

$$
\mathcal{N}(\mathbf{k}, \mathbf{q})=\sum_{n=-\infty}^{\infty} \int_{-\infty}^{\infty} d \nu C_{\nu, n}(Y) F_{\nu, n}(k, q)
$$

where the coefficient function $C_{\nu, n}(Y)$ is found below plugging eq. (2.10) in the BK equation. Here we make a basic assumption that the coefficient function $C_{\nu, n}(Y)$ does not depend on $k$ or $q$. We discuss this approximation in more details below, where we consider the initial condition and the high energy limit of the obtained BK solution.

It is useful to introduce new complex variables

$$
\frac{k}{k-q}=w, \frac{k^{*}}{k^{*}-q^{*}}=w^{*} .
$$

In terms of the coordinates $w$ and $w^{*}$ the function in eq. (2.7) reads

$$
F_{\nu, n}(k, q)=\tilde{F}_{\nu, n}(w, q)=\frac{1}{\sqrt{2} \pi} \frac{|w-1|^{2}}{|q|^{2}} w^{i \nu+\frac{n}{2}-\frac{1}{2}} w^{* i \nu-\frac{n}{2}-\frac{1}{2}}
$$

and the completeness relation in eq. (2.9) is given by

$$
\sum_{n=-\infty}^{\infty} \int_{-\infty}^{\infty} d \nu \tilde{F}_{\nu, n}^{*}\left(w^{\prime}, q\right) \tilde{F}_{\nu, n}(w, q)=\frac{|w-1|^{4}}{|q|^{4}} \delta^{2}\left(w-w^{\prime}\right)
$$

Consider the first linear term of the BK equation in the momentum space eq. (2.5)

$$
\frac{\bar{\alpha}_{s}}{2 \pi} \int \frac{d^{2} \mathbf{k}^{\prime}}{\left(\mathbf{k}-\mathbf{k}^{\prime}\right)^{2}} \mathcal{N}\left(\mathbf{k}^{\prime}, \mathbf{q}\right)=\frac{\bar{\alpha}}{2 \pi} \sum_{n=-\infty}^{\infty} \int_{-\infty}^{\infty} d \nu C_{\nu, n}(Y) \int \frac{d^{2} \mathbf{k}^{\prime}}{\left(\mathbf{k}-\mathbf{k}^{\prime}\right)^{2}} F_{\nu, n}\left(k^{\prime}, q\right)
$$

and calculate the following expression

$$
\begin{aligned}
\int \frac{d^{2} \mathbf{k}^{\prime}}{\left(\mathbf{k}-\mathbf{k}^{\prime}\right)^{2}} F_{\nu, n}\left(k^{\prime}, q\right) & =\int \frac{d^{2} \mathbf{k}^{\prime}}{\left(\mathbf{k}-\mathbf{k}^{\prime}\right)^{2}} \frac{1}{\sqrt{2} \pi} \frac{1}{\left|k^{\prime}\right|\left|k^{\prime}-q\right|}\left(\frac{k^{\prime}}{k^{\prime}-q}\right)^{i \nu+\frac{n}{2}}\left(\frac{k^{\prime *}}{k^{\prime *}-q^{*}}\right)^{i \nu-\frac{n}{2}} \\
& =\frac{1}{\sqrt{2} \pi} \frac{|w-1|^{2}}{|q|^{2}} \int \frac{d^{2} \mathbf{w}^{\prime}}{\left|w-w^{\prime}\right|^{2}} \frac{w^{\prime i \nu+\frac{n}{2}} w^{\prime * i \nu-\frac{n}{2}}}{\left|w^{\prime}\right|} \\
& =\frac{1}{\sqrt{2} \pi} \frac{|w-1|^{2}}{|q|^{2}} \frac{w^{i \nu+\frac{n}{2}} w^{* i \nu-\frac{n}{2}}}{|w|} \int \frac{d^{2} \mathbf{z} z^{i \nu+\frac{n}{2}-\frac{1}{2}} z^{* i \nu-\frac{n}{2}-\frac{1}{2}}}{|1-z|^{2}} \\
& =F_{\nu, n}(k, q) \int \frac{d^{2} \mathbf{z}}{|1-z|^{2}} z^{i \nu+\frac{n}{2}-\frac{1}{2}} z^{* i \nu-\frac{n}{2}-\frac{1}{2}}
\end{aligned}
$$

with $z=w^{\prime} / w$. Thus we have

$$
\frac{\bar{\alpha}_{s}}{\pi} \int \frac{d^{2} \mathbf{k}^{\prime}}{\left(\mathbf{k}-\mathbf{k}^{\prime}\right)^{2}} \mathcal{N}\left(\mathbf{k}^{\prime}, \mathbf{q}\right)=\frac{\bar{\alpha}_{s}}{\pi} \sum_{n=-\infty}^{\infty} \int_{-\infty}^{\infty} d \nu C_{\nu, n}(Y) F_{\nu, n}(k, q) \int \frac{d^{2} \mathbf{z}}{|1-z|^{2}} z^{i \nu+\frac{n}{2}-\frac{1}{2}} z^{* i \nu-\frac{n}{2}-\frac{1}{2}}
$$


In a similar way the second linear term of the BK equation in eq. (2.10) can be written as

$$
-\frac{1}{4} \frac{\bar{\alpha}_{s}}{\pi} \int \frac{d^{2} \mathbf{k}^{\prime}}{\left(\mathbf{k}-\mathbf{k}^{\prime}\right)^{2}} \frac{(\mathbf{q}-\mathbf{k})^{2}}{\left(\mathbf{q}-\mathbf{k}^{\prime}\right)^{2}} \mathcal{N}(\mathbf{k}, \mathbf{q})=-\frac{1}{4} \frac{\bar{\alpha}_{s}}{\pi} \sum_{n=-\infty}^{\infty} \int_{-\infty}^{\infty} d \nu C_{\nu, n}(Y) F_{\nu, n}(k, q) \int \frac{d^{2} \mathbf{z}}{|1-z|^{2}}
$$

and finally the third linear term reads

$$
-\frac{1}{4} \frac{\bar{\alpha}_{s}}{\pi} \int \frac{d^{2} \mathbf{k}^{\prime}}{\left(\mathbf{k}-\mathbf{k}^{\prime}\right)^{2}} \frac{\mathbf{k}^{2}}{\mathbf{k}^{\prime 2}} \mathcal{N}(\mathbf{k}, \mathbf{q})=-\frac{1}{4} \frac{\bar{\alpha}_{s}}{\pi} \sum_{n=-\infty}^{\infty} \int_{-\infty}^{\infty} d \nu C_{\nu, n}(Y) F_{\nu, n}(k, q) \int \frac{d^{2} \mathbf{z}}{|z|^{2}|1-z|^{2}}
$$

The sum of these three linear terms gives an expression, which determines the BFKL eigenvalue

$$
\omega(\nu, n)=\bar{\alpha}_{s} \chi(\nu, n)
$$

through a function

$$
\begin{aligned}
\chi(\nu, n) & =\int \frac{d^{2} \mathbf{z}}{|1-z|^{2}}\left(z^{i \nu+\frac{n}{2}+\frac{1}{2}} z^{* i \nu-\frac{n}{2}-\frac{1}{2}}-\frac{1}{|z|^{2}+|1-z|^{2}}\right) \\
& =2 \psi(1)-\psi\left(i \nu+\frac{|n|}{2}+\frac{1}{2}\right)-\psi\left(-i \nu+\frac{|n|}{2}+\frac{1}{2}\right),
\end{aligned}
$$

which is expressed in terms of the digamma function defined as a logarithmic derivative of Euler gamma function

$$
\psi(z)=\frac{d \ln \Gamma(z)}{d z}=\int_{0}^{1} d x \frac{x^{z-1}}{x-1} .
$$

In deriving eq. (2.20) we used the identity

$$
\int \frac{d^{2} \mathbf{z}}{|z|^{2}|1-z|^{2}}=2 \int \frac{d^{2} \mathbf{z}}{|1-z|^{2}\left(|z|^{2}+|1-z|^{2}\right)}
$$

For more details on the calculation of $\chi(\nu, n)$ the reader is referred to review texts [18-22]. This way we have shown that $F_{\nu, n}(k, q)$ in eq. (2.7) is indeed the BFKL eigenfunction with the eigenvalue $\omega(\nu, n)$ given in commonly used notation without any possible shift of the argument of digamma functions.

Now we are in position of plugging $F_{\nu, n}(k, q)$ into the BK equation in eq. (2.5). Namely, consider the non-linear term

$$
\begin{aligned}
& -\frac{\bar{\alpha}_{s}}{2 \pi} \int d^{2} \mathbf{k}^{\prime} \mathcal{N}\left(\mathbf{k}, \mathbf{k}^{\prime}\right) \mathcal{N}\left(\mathbf{k}-\mathbf{k}^{\prime}, \mathbf{q}-\mathbf{k}^{\prime}\right) \\
& =-\frac{\bar{\alpha}_{s}}{2 \pi} \int d^{2} \mathbf{k}^{\prime} \sum_{n_{1}=-\infty}^{\infty} \sum_{n_{2}=-\infty}^{\infty} \int_{-\infty}^{\infty} d \nu_{1} \int_{-\infty}^{\infty} d \nu_{2} C_{\nu_{1}, n_{1}}(Y) C_{\nu_{2}, n_{2}}(Y) F_{\nu_{1}, n_{1}}\left(k, k^{\prime}\right) F_{\nu_{2}, n_{2}}\left(k-k^{\prime}, q-k^{\prime}\right) \\
& =-\frac{\bar{\alpha}_{s}}{2 \pi} \sum_{n_{1}=-\infty}^{\infty} \sum_{n_{2}=-\infty}^{\infty} \int_{-\infty}^{\infty} d \nu_{1} \int_{-\infty}^{\infty} d \nu_{2} \frac{k^{i \nu_{1}+\frac{n_{1}}{2}}}{(k-q)^{i \nu_{2}+\frac{n_{2}}{2}}} \frac{k^{* i \nu_{1}-\frac{n_{1}}{2}}}{\left(k^{*}-q^{*}\right)^{i \nu_{2}-\frac{n_{2}}{2}}} \frac{1}{|k||k-q|} C_{\nu_{1}, n_{1}}(Y) C_{\nu_{2}, n_{2}}(Y) R_{\nu_{1}, n_{1}}^{\nu_{2}, n_{2}}
\end{aligned}
$$


where $R_{\nu_{1}, n_{1}}^{\nu_{2}, n_{2}}$ is calculated as follows (here we define $k / k^{*}=e^{i 2 \phi}$ )

$$
\begin{aligned}
R_{\nu_{1}, n_{1}}^{\nu_{2}, n_{2}} & =\int \frac{d^{2} \mathbf{k}^{\prime}}{2 \pi^{2}}\left(k-k^{\prime}\right)^{-i \nu_{1}-\frac{n_{1}}{2}+i \nu_{2}+\frac{n_{2}}{2}-1}\left(k^{*}-k^{\prime *}\right)^{-i \nu_{1}+\frac{n_{1}}{2}+i \nu_{2}-\frac{n_{2}}{2}-1} \\
& =\int_{0}^{\infty} \frac{d\left(\left|k^{\prime}\right|^{2}\right)}{4 \pi^{2}} \int_{0}^{2 \pi} d \phi\left|k^{\prime}\right|^{-i 2 \nu_{1}+i 2 \nu_{2}-2} e^{i \phi\left(n_{2}-n_{1}\right)}=\delta\left(\nu_{2}-\nu_{1}\right) \delta_{n_{1}, n_{2}} .
\end{aligned}
$$

Using the last expression we write

$$
-\frac{\bar{\alpha}_{s}}{2 \pi} \int d^{2} \mathbf{k}^{\prime} \mathcal{N}\left(k, k^{\prime}\right) \mathcal{N}\left(k-k^{\prime}, q-k^{\prime}\right)=-\frac{\bar{\alpha}_{s}}{\sqrt{2}} \sum_{n=-\infty}^{\infty} \int_{-\infty}^{\infty} d \nu F_{\nu, n}(k, q)\left(C_{\nu, n}(Y)\right)^{2}
$$

Note that the coefficient of the non-linear term typically associated with the triple Pomeron vertex, in this notation, is a number independent of anomalous dimension and conformal spin. Therefore the $(\nu, n)$ dependence of the BK solution enters only through eigenfunctions $F_{\nu, n}(k, q)$ and the BFKL eigenvalue $\omega(\nu, n)$ in eq. (2.7) and eq. (2.19) respectively.

Using the expression for the non-linear term eq. (2.26) in the BK equation we get an equation for the coefficient function $C_{\nu, n}(Y)$ as follows

$$
\frac{d C_{\nu, n}(Y)}{d Y}=a C_{\nu, n}(Y)+b C_{\nu, n}(Y)^{2} .
$$

Its solution reads

$$
C_{\nu, n}(Y)=\frac{a C_{\nu, n}(0) e^{a Y}}{a+b C_{\nu, n}(0)\left(1-e^{a Y}\right)},
$$

where $a=\omega(\nu, n)$ is the BFKL eigenvalue in eq. $(2.19)$ and $b=-\overline{\alpha_{s}} /(\sqrt{2})$ is the coefficient of the non-linear term in eq. (2.26). ${ }^{2}$ The initial condition of the coefficient function $C_{\nu, n}(Y)$ is set by the following expression

$$
C_{\nu, n}(0)=|q|^{2} \int d^{2} k^{\prime} F_{\nu, n}^{*}\left(k^{\prime}, q\right) \Phi\left(k^{\prime}, q\right)=|q|^{4} \int \frac{d^{2} w^{\prime}}{\left|w^{\prime}-1\right|^{4}} \tilde{F}_{\nu, n}^{*}\left(w^{\prime}, q\right) \tilde{\Phi}\left(w^{\prime}, q\right) .
$$

The function $\Phi(k, q)$ is some impact factor determining an initial condition of the non-linear evolution. The self-consistency of the approach suggests a choice of the impact factor describing multiple rescatterings, e.g. an impact factor of the Glauber-Mueller type [32] (see also ref. [33]). Our basic assumption is that the coefficient function $C_{\nu, n}(0)$ does not depend on the transferred momentum $q$. This means that we choose the impact factor in such a way that

$$
\tilde{\Phi}\left(w^{\prime}, q\right)=\frac{\tilde{\Phi}\left(w^{\prime}\right)}{|q|^{2}}
$$

in accordance with eq. (2.29) and eq. (2.12), where $\tilde{\Phi}(w)$ is some function of $w$ and independent of $q$. This approximation seems to be reasonable for small values of the transferred momentum.

\footnotetext{
${ }^{2}$ Note that this coefficient depends on the normalization of the eigenfunctions.
} 
The evolution equation eq. (2.27) and its solution in eq. (2.28) are similar to that of the phenomenological summation of the Pomeron Fan diagrams in the Reggen Field Theory for zero transverse momentum $[34,35]$ and can be used for summation of pomeron loops [36-40]. We recall that we wrote a general form of the BK solution in eq. (2.10) as

$$
\mathcal{N}(\mathbf{k}, \mathbf{q})=\sum_{n=-\infty}^{\infty} \int_{-\infty}^{\infty} d \nu C_{\nu, n}(Y) F_{\nu, n}(k, q),
$$

and it follows directly from this definition and the completeness condition in eq. (2.9) that at $Y=0$ for our choice of $C_{\nu, n}(Y)$ we have

$$
\left.\mathcal{N}(\mathbf{k}, \mathbf{q})\right|_{Y=0}=\Phi(\mathbf{k}, \mathbf{q}) .
$$

A general form of the solution $\mathcal{N}(\mathbf{k}, \mathbf{q})$ in eq. (2.31) together with the expression for the coefficient function $C_{\nu, n}(Y)$ in eq. (2.28) and its initial condition $C_{\nu, n}(0)$ in eq. (2.29) present the main result of the this study.

It is easy to see from eq. (2.10) and eq. (2.28) that the solution of the BFKL equation in the color singlet state is obtained by setting $b=0$ in the coefficient function $C_{\nu, n}(Y)$. Note that in the BFKL equation, which corresponds to the linear part of eq. (2.5) the transferred momentum $q$ is not mixed with the integration variable of the Kernel and thus the condition that $C_{\nu, n}(Y)$ does not depend on $q$ can be relaxed reproducing the full analytic solution of the BFKL equation for a non-zero transferred momentum.

We have shown above that our choice of functions $F_{\nu, n}(k, q)$ is consistent with a solution of the BFKL equation, and the next step is to check that the full solution written in terms of these functions have a reasonable behaviour at high energy.

In the limit $Y \rightarrow \infty$ eq. (2.28) reduced to

$$
C_{\nu, n} \simeq-\frac{a}{b}=\sqrt{2} \chi(\nu, n)
$$

and the amplitude $\mathcal{N}(\mathbf{k}, \mathbf{q})$ defined by eq. (2.10) in this limit does not depend of the initial condition resulting in

$$
\left.\mathcal{N}(\mathbf{k}, \mathbf{q})\right|_{Y \rightarrow \infty} \simeq \sum_{n=-\infty}^{\infty} \int_{-\infty}^{\infty} d \nu \sqrt{2} \chi(\nu, n) F_{\nu, n}(k, q)=\frac{2}{|q|^{2}}
$$

Details of this calculation are presented in the appendix. This function peaks at small $q$ and thus can be reasonably approximated by Dirac $\delta$-function.

$$
\left.\mathcal{N}(\mathbf{k}, \mathbf{q})\right|_{Y \rightarrow \infty} \simeq 2 \delta\left(|q|^{2}\right) \delta(\phi)=\frac{1}{|q|} \delta(|q|) \delta(\phi)=\delta^{2}(\mathbf{q}) .
$$

Note that we have no $k$ dependence at $Y \rightarrow \infty$ and the asymptotic amplitude diverges for $q=0$. This amplitude represents so called fixed point at which the derivative $\partial_{Y} \mathcal{N}(k, q)$ vanishes and the amplitude goes to its maximal value. On the other hand one can search fixed points of the BK equation in the coordinate space in eq. (2.1) and it is widely accepted that a natural fixed point of eq. (2.1) is $N(\mathbf{x}, \mathbf{y})=1$, which is also a fixed point in 
phenomenological models. Then for our definition of the Fourier transform in eq. (2.4) we have

$$
\begin{aligned}
\mathcal{N}(\mathbf{k}, \mathbf{q}) & =\int \frac{d^{2} \mathbf{x}}{2 \pi} \frac{d^{2} \mathbf{y}}{2 \pi} e^{i \mathbf{k x}} e^{i(\mathbf{q}-\mathbf{k}) \mathbf{y}} \frac{\theta\left((\mathbf{x}-\mathbf{y})^{2}-c^{2}\right)}{(\mathbf{x}-\mathbf{y})^{2}} \\
& =-\frac{\delta^{2}(\mathbf{q})}{2}\left(\ln \left(\frac{\mathbf{k}^{2}}{4}\right)-2 \psi(1)+\ln c^{2}\right)
\end{aligned}
$$

We have a certain freedom in choosing the cut-off $c$, which we fix by imposing a condition that the expressions in eq. (2.35) and eq. (2.36) are to be identical. This condition imposes a definite choice of the cut-off such that

$$
c^{2}=\frac{4 e^{2 \psi(1)-2}}{k^{2}} .
$$

Note that this choice of the cut-off can be related to two basic values that govern the saturation physics. Namely, the coefficient that determines the rapidity dependence of the saturation scale ${ }^{3}$

$$
Q_{s}(Y) \simeq Q_{s}(0) e^{\bar{\alpha}_{s} \frac{C}{2} Y}, C \simeq 4.88
$$

and the critical exponent $\gamma_{c}=0.6275$ in

$$
N(\mathbf{x}, \mathbf{y}) \propto|\mathbf{x}-\mathbf{y}|^{2 \gamma_{c}} \ln |\mathbf{x}-\mathbf{y}|, \quad \text { for } \quad|\mathbf{x}-\mathbf{y}| \rightarrow 0 .
$$

Those two can be expressed at one percent accuracy through our choice of the cut-off as follows

$$
\frac{2}{\sqrt{c^{2} k^{2}}} \simeq 4.84
$$

and

$$
\frac{1}{\ln \left(\frac{2}{\sqrt{c^{2} k^{2}}}\right)} \simeq 0.634
$$

establishing a relation

$$
\frac{1}{\ln C} \simeq \gamma_{c}
$$

The authors are not aware about any previous study discussing a similar relation.

\section{Discussions}

We discuss a new form of the singlet BFKL eigenfunction in eq. (2.7) and using it build an analytic solution of the BK equation in eq. (2.10) for a particular kinematics, where the impact factor depends on the transferred momentum through eq. (2.30). We check that the obtained solution satisfies the initial condition, it has a proper high energy behavior compatible with known values of the saturation scale and its form resembles a solution to phenomenological fan diagrams resummation.

\footnotetext{
${ }^{3}$ For most recent review on saturation physics the reader is refrerred to the book of Yu. V. Kovchegov and E. M. Levin and other review texts [18-22].
} 
The Balitsky-Kovchegov equation was extensively studied during past two decades both numerically and analytically in various approximations. The comparison of the solution presented in this paper to the approximate solutions available in the literature is not a simple task and definitely requires a separate analysis which will be published by us elsewhere. The most important cross check for us is the compatibility with the linear evolution of the BFKL equation and the proper high energy behaviour as shown above. We expect some peculiar technicalities in going back to the coordinate space we have started with in eq. (2.1), related to the fact that we are dealing with individually divergent quantities, which nevertheless result into a finite and well defined final answer. The proposed analytic solution of the BK equation may have many phenomenological applications [41, 42], e.g. effective high energy description of the proton-nucleus scattering as well as nucleus-nucleus scattering calculated through pomeron loops built of two symmetric (projectile-target) BK solutions.

The singlet BFKL equation is reach of symmetries [43-47] and being integrable is supposed to enjoy also the dual conformal symmetry as mentioned above. A similar though a more general dual superconformal conformal symmetry was identified for planar scattering amplitudes in maximally supersymmetric theory (see recent results in ref. [48] and references wherein). The solution of the Balitsky-Kovchegov equation presented here is written in the space of transverse momenta and one can expect some manifistations of the dual superconformal symmetry appearing also in the case of the non-linear BK evolution. We leave these issues for our further publications.

\section{Acknowledgments}

The authors are indebted to I. Balitsky, J. Bartels, M. Braun, E. Gotsman, G. Korchemsky, E. Levin and L. Lipatov for enlightening discussions.

Special thanks goes to J. Bartels for his warm hospitality during a stay of A.P. at University of Hamburg/DESY, where this project was initiated. The work of A.P. on this paper was supported in part by SFB-676 Fellowship of Deutsche Forschungsgemeinschaft (DFG).

\section{A Calculation of $\left.\mathcal{N}(\mathrm{k}, \mathrm{q})\right|_{Y \rightarrow \infty}$}

We calculate the high energy asymptotics of the solution in eq. (2.31)

$$
\left.\mathcal{N}(\mathbf{k}, \mathbf{q})\right|_{Y \rightarrow \infty} \simeq \sum_{n=-\infty}^{\infty} \int_{-\infty}^{\infty} d \nu \sqrt{2} \chi(\nu, n) F_{\nu, n}(k, q),
$$

where the function $\chi(\nu, n)$ is given in eq. (2.20) by

$$
\begin{aligned}
\chi(\nu, n) & =2 \psi(1)-\psi\left(i \nu+\frac{|n|}{2}+\frac{1}{2}\right)-\psi\left(-i \nu+\frac{|n|}{2}+\frac{1}{2}\right) \\
& =-\sum_{m=1}^{\infty} \frac{i \nu+\frac{|n|}{2}-\frac{1}{2}}{m\left(m+i \nu+\frac{|n|}{2}-\frac{1}{2}\right)}-\sum_{m=1}^{\infty} \frac{-i \nu+\frac{|n|}{2}-\frac{1}{2}}{m\left(m-i \nu+\frac{|n|}{2}-\frac{1}{2}\right)}
\end{aligned}
$$


and the eigenfunction $F_{\nu, n}(k, q)$ is defined in eq. (2.14) as follows

$$
F_{\nu, n}(k, q)=\tilde{F}_{\nu, n}(w, q)=\frac{1}{\sqrt{2} \pi} \frac{|w-1|^{2}}{|q|^{2}} w^{i \nu+\frac{n}{2}-\frac{1}{2}} w^{* i \nu-\frac{n}{2}-\frac{1}{2}} .
$$

Consider the following expression

$$
\begin{aligned}
I & =\sum_{n=-\infty}^{\infty} \int_{-\infty}^{\infty} d \nu \chi(\nu, n) w^{i \nu+\frac{n}{2}} w^{* i \nu-\frac{n}{2}}=\sum_{n=-\infty}^{\infty} \int_{-\infty}^{\infty} d \nu \chi(\nu, n)|w|^{i 2 \nu} e^{i n \phi} \\
& =\sum_{n=1}^{\infty} \int_{-\infty}^{\infty} d \nu \chi(\nu, n)|w|^{i 2 \nu}\left(e^{i n \phi}+e^{-i n \phi}\right)+\int_{-\infty}^{\infty} d \nu \chi(\nu, 0)|w|^{i 2 \nu}
\end{aligned}
$$

where we use $w / w^{*}=e^{i \phi}$. We do the $\nu$ integration first for $|w|>1$ closing integration contour in the upper complex semiplane and the contribution comes only from one of the digamma functions in $\chi(\nu, n)$. Namely,

$$
\begin{aligned}
I & =2 \pi \sum_{m=1}^{\infty} \sum_{n=1}^{\infty}|w|^{1-n-2 m}\left(e^{i n \phi}+e^{-i n \phi}\right)+2 \pi \sum_{m=1}^{\infty}|w|^{1-2 m} \\
& =2 \pi \sum_{m=1}^{\infty}|w|^{1-2 m}\left(1+\sum_{n=1}^{\infty}\left(w^{*-n}+w^{-n}\right)\right) \\
& =2 \pi \frac{|w|}{|w|^{2}-1}\left(\frac{1}{w^{*}-1}+\frac{1}{w-1}+1\right)=2 \pi \frac{|w|}{|w-1|^{2}} .
\end{aligned}
$$

In a similar way we close the integration contour for $\nu$ in the lower complex semiplane for $|w|<1$ and get the same result. Using the definition of $F_{\nu, n}(k, q)$ in eq. (2.14) we finally obtain

$$
\left.\mathcal{N}(\mathbf{k}, \mathbf{q})\right|_{Y \rightarrow \infty} \simeq \sum_{n=-\infty}^{\infty} \int_{-\infty}^{\infty} d \nu \sqrt{2} \chi(\nu, n) F_{\nu, n}(k, q)=\frac{|w-1|^{2}}{\pi|q|^{2}|w|} I=\frac{2}{|q|^{2}} .
$$

Open Access. This article is distributed under the terms of the Creative Commons Attribution License (CC-BY 4.0), which permits any use, distribution and reproduction in any medium, provided the original author(s) and source are credited.

\section{References}

[1] L.N. Lipatov, Reggeization of the vector meson and the vacuum singularity in non-Abelian gauge theories, Sov. J. Nucl. Phys. 23 (1976) 338 [Yad. Fiz. 23 (1976) 642] [InSPIRE].

[2] E.A. Kuraev, L.N. Lipatov and V.S. Fadin, The Pomeranchuk singularity in non-Abelian gauge theories, Sov. Phys. JETP 45 (1977) 199 [Zh. Eksp. Teor. Fiz. 72 (1977) 377] [INSPIRE].

[3] I.I. Balitsky and L.N. Lipatov, The Pomeranchuk singularity in quantum chromodynamics, Sov. J. Nucl. Phys. 28 (1978) 822 [Yad. Fiz. 28 (1978) 1597] [INSPIRE].

[4] L.V. Gribov, E.M. Levin and M.G. Ryskin, Semihard processes in $Q C D$, Phys. Rept. 100 (1983) 1 [INSPIRE]. 
[5] I. Balitsky, Operator expansion for high-energy scattering, Nucl. Phys. B 463 (1996) 99 [hep-ph/9509348] [INSPIRE].

[6] Y.V. Kovchegov, Small-x $F_{2}$ structure function of a nucleus including multiple Pomeron exchanges, Phys. Rev. D 60 (1999) 034008 [hep-ph/9901281] [INSPIRE].

[7] Y.V. Kovchegov, Unitarization of the BFKL Pomeron on a nucleus, Phys. Rev. D 61 (2000) 074018 [hep-ph/9905214] [inSPIRE].

[8] N.N. Nikolaev and B.G. Zakharov, Color transparency and scaling properties of nuclear shadowing in deep inelastic scattering, Z. Phys. C 49 (1991) 607 [InSPIRE].

[9] N.N. Nikolaev and B.G. Zakharov, On determination of the large $1 / x$ gluon distribution at HERA, Phys. Lett. B 332 (1994) 184 [hep-ph/9403243] [INSPIRE].

[10] A.H. Mueller, Soft gluons in the infinite momentum wave function and the BFKL Pomeron, Nucl. Phys. B 415 (1994) 373 [inSPIRE].

[11] A.H. Mueller and B. Patel, Single and double BFKL Pomeron exchange and a dipole picture of high-energy hard processes, Nucl. Phys. B 425 (1994) 471 [hep-ph/9403256] [INSPIRE].

[12] A.H. Mueller, Unitarity and the BFKL Pomeron, Nucl. Phys. B 437 (1995) 107 [hep-ph/9408245] [INSPIRE].

[13] Z. Bern, L.J. Dixon and V.A. Smirnov, Iteration of planar amplitudes in maximally supersymmetric Yang-Mills theory at three loops and beyond, Phys. Rev. D 72 (2005) 085001 [hep-th/0505205] [INSPIRE].

[14] L. Lipatov, A. Prygarin and H.J. Schnitzer, The multi-Regge limit of NMHV amplitudes in $N=4$ SYM theory, JHEP 01 (2013) 068 [arXiv:1205.0186] [INSPIRE].

[15] V.S. Fadin, R. Fiore, L.N. Lipatov and A. Papa, Moebius invariant BFKL equation for the adjoint representation in $N=4$ SUSY, arXiv:1305.3395 [INSPIRE].

[16] V.S. Fadin, R. Fiore, L.N. Lipatov and A. Papa, Mobius invariant BFKL equation for the adjoint representation in $N=4$ SUSY, Nucl. Phys. B 874 (2013) 230 [InSPIRE].

[17] C. Marquet and G. Soyez, The Balitsky-Kovchegov equation in full momentum space, Nucl. Phys. A 760 (2005) 208 [hep-ph/0504080] [INSPIRE].

[18] L.N. Lipatov, Small-x physics in perturbative QCD, Phys. Rept. 286 (1997) 131 [hep-ph/9610276] [INSPIRE].

[19] V. Barone and E. Predazzi, High-energy particle diffraction, Springer, Germany (2002) [INSPIRE].

[20] J. Forshaw and D. Ross, Quantum chromodynamics and the Pomeron, Cambridge Lecture Notes in Physics, Cambridge University Press, Cambridge U.K. (1997) [InSPIRE].

[21] B.L. Ioffe, V.S. Fadin and L.N. Lipatov, Quantum chromodynamics: perturbative and nonperturbative aspects, Cambridge University Press, Cambridge U.K. (2014) [INSPIRE].

[22] Yu.V. Kovchegov and E.M. Levin, Quantum chromodynamics at high energy, Cambridge University Press, Cambridge U.K. (2012) [INSPIRE].

[23] J. Bartels, L.N. Lipatov and A. Sabio Vera, $N=4$ supersymmetric Yang-Mills scattering amplitudes at high energies: the Regge cut contribution, Eur. Phys. J. C 65 (2010) 587 [arXiv:0807.0894] [INSPIRE]. 
[24] L.N. Lipatov and A. Prygarin, Mandelstam cuts and light-like Wilson loops in $N=4$ SUSY, Phys. Rev. D 83 (2011) 045020 [arXiv: 1008. 1016] [InSPIRE].

[25] L.N. Lipatov and A. Prygarin, BFKL approach and six-particle MHV amplitude in $N=4$ super Yang-Mills, Phys. Rev. D 83 (2011) 125001 [arXiv:1011.2673] [INSPIRE].

[26] J. Bartels, L.N. Lipatov and A. Prygarin, $M H V$ amplitude for $3 \rightarrow 3$ gluon scattering in Regge limit, Phys. Lett. B 705 (2011) 507 [arXiv: 1012.3178] [InSPIRE].

[27] J. Bartels, L.N. Lipatov and A. Prygarin, Integrable spin chains and scattering amplitudes, J. Phys. A 44 (2011) 454013 [arXiv:1104.0816] [inSPIRE].

[28] J. Bartels, L.N. Lipatov and A. Prygarin, Collinear and Regge behavior of $2 \rightarrow 4 \mathrm{MHV}$ amplitude in $N=4$ super Yang-Mills theory, arXiv:1104.4709 [INSPIRE].

[29] A. Prygarin, M. Spradlin, C. Vergu and A. Volovich, All two-loop MHV amplitudes in multi-Regge kinematics from applied symbology, Phys. Rev. D 85 (2012) 085019 [arXiv: 1112.6365] [INSPIRE].

[30] J. Bartels, A. Kormilitzin, L.N. Lipatov and A. Prygarin, BFKL approach and $2 \rightarrow 5$ maximally helicity violating amplitude in $N=4$ super-Yang-Mills theory, Phys. Rev. D 86 (2012) 065026 [arXiv:1112.6366] [INSPIRE].

[31] G. Chachamis and A.S. Vera, The NLO N=4 SUSY BFKL Green function in the adjoint representation, Phys. Lett. B 717 (2012) 458 [arXiv:1206.3140] [INSPIRE].

[32] A.H. Mueller, Small-x behavior and parton saturation: a QCD model, Nucl. Phys. B 335 (1990) 115 [inSPIRE].

[33] S. Bondarenko and M.A. Braun, Boundary conditions in the QCD nucleus-nucleus scattering problem, Nucl. Phys. A 799 (2008) 151 [arXiv:0708.3629] [INSPIRE].

[34] S. Bondarenko, E. Gotsman, E. Levin and U. Maor, A Pomeron approach to hadron nucleus and nucleus-nucleus 'soft' interactions at high-energy, Nucl. Phys. A 683 (2001) 649 [hep-ph/0001260] [INSPIRE].

[35] S. Bondarenko, L. Motyka, A.H. Mueller, A.I. Shoshi and B.-W. Xiao, On the equivalence of Reggeon field theory in zero transverse dimensions and reaction-diffusion processes, Eur. Phys. J. C 50 (2007) 593 [hep-ph/0609213] [InSPIRE].

[36] M. Kozlov, E. Levin and A. Prygarin, The BFKL Pomeron calculus: probabilistic interpretation and high energy amplitude, hep-ph/0606260 [INSPIRE].

[37] E. Levin and A. Prygarin, The BFKL Pomeron calculus in zero transverse dimension: summation of the Pomeron loops and the generating functional for the multiparticle production processes, Eur. Phys. J. C 53 (2008) 385 [hep-ph/0701178] [INSPIRE].

[38] M. Kozlov, E. Levin and A. Prygarin, The BFKL Pomeron calculus in the dipole approach, Nucl. Phys. A 792 (2007) 122 [arXiv:0704.2124] [INSPIRE].

[39] M. Kozlov, E. Levin and A. Prygarin, The BFKL Pomeron calculus in the dipole approach, Nucl. Phys. A 792 (2007) 122 [arXiv:0704.2124] [INSPIRE].

[40] E. Levin, J. Miller and A. Prygarin, Summing Pomeron loops in the dipole approach, Nucl. Phys. A 806 (2008) 245 [arXiv:0706.2944] [InSPIRE].

[41] J. Bartels, S. Bondarenko, K. Kutak and L. Motyka, Exclusive Higgs boson production at the LHC: hard rescattering corrections, Phys. Rev. D 73 (2006) 093004 [hep-ph/0601128] [INSPIRE]. 
[42] S. Bondarenko and L. Motyka, Solving effective field theory of interacting QCD Pomerons in the semi-classical approximation, Phys. Rev. D 75 (2007) 114015 [hep-ph/0605185] [INSPIRE].

[43] L.N. Lipatov, Duality symmetry of Reggeon interactions in multicolor QCD, Nucl. Phys. B 548 (1999) 328 [hep-ph/9812336] [INSPIRE].

[44] A. Prygarin, Duality symmetry in high energy scattering, arXiv:0908.2386 [INSPIRE].

[45] A. Prygarin, Duality symmetry of BFKL equation: reggeized gluons versus color dipoles, Phys. Rev. C 83 (2011) 055206 [arXiv:0911.5279] [InSPIRE].

[46] C. Gomez, J. Gunnesson and A.S. Vera, Dual conformal invariance in the Regge limit, Phys. Lett. B 690 (2010) 78 [arXiv:0908.2568] [INSPIRE].

[47] S. Bondarenko and A. Prygarin, BFKL ansatz for BK equation in conformal basis, Nucl. Phys. A 800 (2008) 63 [arXiv:0709.3010] [InSPIRE].

[48] S. Derkachov, G.P. Korchemsky and A.N. Manashov, Dual conformal symmetry on the light-cone, Nucl. Phys. B 886 (2014) 1102 [arXiv:1306.5951] [INSPIRE]. 